\title{
Design and Research of Training Platform for Computer Skills of Rural Labor Force
}

\author{
Chunhua Lu ${ }^{1,}$, Xinhua $\mathrm{Lu}^{2}$, Fusong Chen ${ }^{3}$ \\ ${ }^{1}$ Department of Physics and Electronic Science, Anshun University, Anshun City, China \\ ${ }^{2}$ No.1 Middle School of Dongming County, Heze City, China \\ ${ }^{3}$ Anshun Municipal Committee Office, Anshun City, China \\ Email address: \\ 1306038381@qq.com (Chunhua Lu),1183287401@qq.com (Xinhua Lu),56260798@qq.com (Fusong Chen) \\ ${ }^{*}$ Corresponding author
}

\section{To cite this article:}

Chunhua Lu, Xinhua Lu, Fusong Chen. Design and Research of Training Platform for Computer Skills of Rural Labor Force. International Journal of Intelligent Information Systems. Vol. 6, No. 3, 2017, pp. 36-39. doi: 10.11648/j.ijiis.20170603.12

Received: June 6, 2017; Accepted: July 4, 2017; Published: August 2, 2017

\begin{abstract}
Rural labor is an important force in the construction course of new socialist countryside, and the level of rural labor skills directly affects the process of new rural construction. This paper aims at the existing issues of computer skills training in rural areas, presents an analysis of the status quo of the old towns, and then describes each functional module design of the computer skills training platform, thus to enhance the skills of the rural labor force, meet the learning needs of the rural labor force, improve the rural labor employment environment, and promote the process of a well-off society.
\end{abstract}

Keywords: Rural Labor Force, Computer Skills, Training Platform

\section{Introduction}

Nowadays, the era of big data has arrived, skills training has encountered unprecedented opportunities and challenges, and good command of computer is becoming increasingly important. Especially in the process of building a well-off society, it's need to fix the bottleneck in terms of computer skills among rural labor force, to improve the competence of rural labor force and their abilities, to keep the rural labor force in this era for "keeping up", thus to promote the sustained and healthy economic and social development. In this context, it is necessary to establish a sound training platform for rural labor computer skills. In this paper, Jiuzhou Town of Xixiu District of Anshun city in Guizhou Province is used as an example, to discuss the computer skills training for rural labor force, with a purpose to provide a useful reference for enhancing the computer skills of rural labor force.

Jiuzhou is an old town under the jurisdiction of Anshun City in Guizhou Province. By the end of 2016, this town has a total population of 46,202 with agricultural population of 42,100 , accounting for $93.14 \%$ of the total [1]. For the economic and social development, its agricultural population is the majority, which is the main force to promote the development of rural areas, however, by solely relying on improving the income of the masses of farmers, especially middle-aged and young migrant farmers is not enough. In this context, computer skills training emerges as a subject that the era requires, but in actual training, the problems such as high cost, poor effectiveness and weak application and so on have seriously dampened the enthusiasm of the rural labor force. How to solve these problems and enhance the entrepreneurship and development ability of rural labor force and aid farmers to increase income and become rich, is particularly important.

\section{Main Body}

(1) Introduction of the development status quo of Jiuzhou Town

Jiuzhou is a national historical and cultural town, with natural ecological resources richly endowed by nature. First of all, it has flat and fertile land with arable area of 2292.9 hectares, it is a typical and traditional agricultural town. Agriculture has been its main business for a long time, and it is known as "Rice Warehouse" of Anshun [2]. There are a great variety of agricultural products, such as high-quality 
yam, houttuynia and rice. But most farmers are relying on their own experience and predecessor experience to grow crops, the yield cannot go high, new planting techniques cannot be applied and promoted, and high-quality agricultural products cannot be sold timely, thus farmers' income is not high, which restricts the improvement of agricultural efficiency.

Secondly, Jiuzhou has folkways cultural resources and unique tourism conditions richly endowed by nature, it has integrated "Jiangnan" elements and Tunpu Culture, forming a unique style of Tunpu and mountain scenery of Jiangnan [3], and it has high historical, scientific and artistic values. Enjoying such abundant resources, Jiuzhou has been actively exploring effective ways for development, but its propaganda of itself to the outside world is not ideal, so many excellent resources have not be been widely known, such as a large number of specialty products are only sold locally, even there are outside orders, they are just small orders with limited sales volume, therefore, the supply and demand have not been balanced. If the rural labor force can master computer technologies and produce sales and advertisement type websites for local tourism resources and cultural resources and issue videos for specialty agricultural products, it would be a qualitative change to propagandize the folk customs to the outside world, to roll out the local specialty products, to establish a good brand image and ensure quality assurance and service, and to promote the sales of local resources. And it will be a fundamental change for the production and sales of local resources.

(2) Training analysis of rural labor skills

With the arrival of the era of big data, Guizhou uses the e-commerce as the way of achieving values by taking shortcut, and the strategic choice for catching up and surpass, it places the "three rural issues" as the main battlefield for the development of e-commerce, the main force and the main channel [4]. In the implementation course of the rural labor transfer project, the training for labor skills should be taken as the key link to promote the transfer of labor force. Facing this golden opportunity for development, Jiuzhou implements the system of "Free Education Voucher for Farmer Skills Training". Any rural farmer who has learning needs can be enrolled for the study, the purpose is to make the farmers to master computer knowledge and develop skills, to facilitate the employment, thus to promote the transfer of labor force. However, this "pro-people project", due to that a large number of rural labor force view the training as time-consuming and laborious, and there is no clear understanding of how to improve their skills, therefore the purpose of training is not clear, it ended up with an embarrassing situation where "the government is enthusiastic, but farmers are indifferent", the learning effect is not ideal, resulting in that the skills training did not generate its due effect. To some extent this has caused the waste of manpower and material resources. In order to reduce the local government's financial burden, provide guidance and service for learning for the rural labor force, and save the cost, this paper will focus on the relation between the delivery of curriculum content and the development of rural labor, meanwhile, it provides the planting technologies for the rural labor force with the option of independent on-line free learning, to solve their problem of energy and time shortage, thus to achieve the independence of work and study.

(3) Design and research of skills training platform for rural labor force

(a) Training course design

The training curriculum resources are the core of the platform construction, and how to present the training content properly is related to the whole operation of the platform. Therefore, during the design of the curriculum resources, Ajax encapsulated with jQuery is used to request data, Javascript is used for dynamic rendering of data, as the different course content is arranged in different JSON data documents, so, in the process of requesting data, a large number of Ajax data will be sent to the server, although you can achieve the same effect, the overall operation smoothness of the platform will be greatly reduced, at the same time, the client browsers will occupy relatively large amount of resources, so the design will need to package the content of requested data as a separate function, using only one Ajax to request, through different functions to transmit different parameters to the same Ajax, so it can save a lot of redundant codes, to reduce unnecessary Ajax requests, and greatly improve the stability and smoothness of platform. After the data transmission approach is designed, the training content is developed based on the characteristics of rural labor and the curriculum features; it is mainly divided into computer basics [5], web front-end development technology, image processing technology, video production and processing, advanced programming basics and so on 5 different sections. Taking into account the education level of farmers, farmers' learning habits, video recording approach is used for various curriculum resources on the platform to develop the course, so that trainees can learn based on their time schedule in non-real time manner by watching the video [6]. In order to allow the trainees to develop a comprehensive grasp of the curriculum, the curriculum is developed by Microsoft Word, making clear introduction to the course learning objectives, learning requirements and time schedule; for the practice part, operations of key parts based on chapters are described in details, in order to help rural labor force with consolidation and application of curriculum knowledge. The course of agricultural technology is mainly about the agricultural growing and animal production techniques, and the links of related agricultural learning websites are added for reference to the rural labor force [7]. The specific presentation for training content on the platform is independent access to web content, triggering navigation menu by mouse to guide rural labor force, such as the Web front-end development technologies, all the sub-menu, HTML, CSS, Javascript, JQuery, domain name and website and so on sub-menus are all concealed before clicking them, when the mouse moves into the area of the corresponding column, their hidden state is visible, and position processing happen by the corresponding anchor tag, if you click the event and it will 
trigger to navigate to the location; this treatment will help the rural labor force to find their own learning resources, to reduce number of clicks, save the search time, and improve browsing efficiency.

(b) Function module design of platform

The functional modules of computer training platform adopt HTM 5+CSS 3 structure for the page layout and style design and JavaScript + jQuery for page dynamic effect and data exchange, JSON data format is used to store the information related to the course and the Java and Mysql database is used to build a server thus to for a complete background end. The functions of the training platform not only focus on the design of training content, but are also reflected in the user's personal registration, online communication, background management, and it highlights stable, practical and convenient features [8].

(i) Log-in and registration module of platform

The registration platform adopts independent panel to edit a single page, the main detail work is for reasonable verification of information, the verification and field parameters are shown in Table 1; to submit registration information will require the above parameters for logic verification, the verification tool used is regular expression, after the registration, the log-in account information will be cached locally and support the exit operation. The following table provides the main parameters for verification (Y: required, $\mathrm{N}$ : optional):

Table 1. Registration verification.

\begin{tabular}{lllll}
\hline Field & User & Password & Phone & Email \\
\hline Parameter & userName & passWord & telNumber & emailCon \\
Required & Y & Y & Y & Y \\
\hline
\end{tabular}

After verification, the registration button becomes available, the information is saved, and then it is linked to the LOGIN panel. The information of successful login user is shown on the home page of the website. With legal account, farmers can browse, modify and search web pages, but they can not update web content, delete or publish information.

(ii) Search module

The platform loads the search results according to the keywords of Baidu search engine by searching the dynamic status of keywords, in order to ensure that the display effect is beautiful, the search box column adopts left-right layout, the most left is the search icon for positioning, the left distance of the search content uses padding attribute to setup, the purpose is to ensure the content does not overlap with the search icon. The right-hand side uses button to make the search button and to add search event behavior.

The ability of the computer farmers is not enough, and they are not familiar with the search engine, when they have problems, they can directly use the search function which saves time.

(iii) Video teaching module

Video teaching is the core design of the platform. The design idea is to separate each content module into an area, which will help the rural labor to learn step by step. Because each individual module can read data from different json files to render, it avoids the confusion of the data region, which reflects the module independence of the front-end design.

The specific design details are mainly reflected in the accuracy of data acquisition and rendering, and the order of rendering is determined by the order of data stored in the json file. Because the json file is a direct source of data module, and the only source of data, it can separate the data from the web page, when needed, you can directly change the content of the data file, there is no need to change the page and events, which is easy to maintain. The design style of the video file interface uses CSS 3 for keyframes animation transition, the transition mode also uses the percentage method, from $0 \%$ to $50 \%$, from $50 \%$ to $100 \%$ and so on ways to set up the corresponding effect and time length for animation transition. In order to unify the overall style of the platform, a border and fillet design is used for the outer parent element. When the mouse is moved into the play area, the corresponding element will have a moving effect. In a word, the independent encapsulation of video content allows for call on demand, it can improve the reusing ability of the function, thus this reduces the redundancy of code to a certain extent and improves the overall operation speed of the platform.

The video teaching module is built from the perspective of learning characteristics of rural labor force, as the learning foundation of this group is not solid enough. The module design of the training platform starts with convenient operation, it pays attention to audio-visual learning, and strengthens the basic knowledge. With the help of field training, the rural labor force can eventually use the knowledge acquired to build websites, to produce and release video resources, and thus to achieve the purpose of promoting and selling local specialty products.

(iv) Online message module

Online message module allows the users to publish messages on the platform after their successful log-in, or to reply messages; the administrators can view, publish messages, delete history and bad messages. Figure 1 shows the layout and optimization style of the online message.

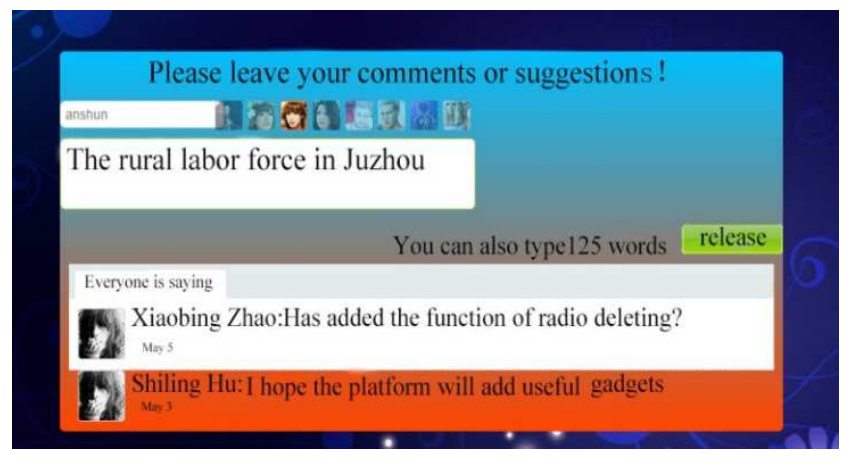

Figure 1. Online messages.

With online messages module, rural labor force will be able to discuss with other trainees or teachers once they encounter any doubt in learning, so as to solve the problem 
[9]; the training teachers then can discover the deficiencies in the teaching according to rural labor force's questions, make the corresponding adjustments to take measures for the shortcomings in the teaching. The website administrator can update the website content and upload training content of different levels according to the learning problems of the rural labor force, the enthusiasm for learning of rural labor force will not be impacted by the learning material level, which can stimulate learning initiatives among them.

(v) Background management module

The background end is built to achieve the rapid operation and management of the database, so that training resources and information will be updated and adjusted in a timely manner. In order to ensure the platform security, this module is only open to the administrators for training content update, add and delete of released information [10], as well as the monitoring of message status, and review of applicant users.

\section{Conclusion}

The proper use of computer skills training platform can not only solve the limit of the rural labor force in terms of learning time and space, but also reduce the training burden of teachers in the field which is convenient for both rural labor force and training teachers, improves the learning effect and stimulates interest in learning; it can effectively improve the computer skill level of rural labor force. At present, the training platform has been initially built. Next, it is need to roll out to the rural labor force. Meanwhile it is hoped to share the Jiuzhou Town experience and training mode, and provide effective reference for other areas for skill training, thus to promote the healthy development of rural economy and society.

\section{Acknowledgements}

(1) Three-Party Collaborative Fund of Guizhou Province, Anshun government and Anshun University. Project Name: Design Study of Computer Skills Training Platform. Contract No.: QKHLHZ [2015]7701.
(2) Teaching Content and Curriculum System Reform Project of Higher Education Institutions of Guizhou Province, Project Name: NCRE Based Revolutionary Exploration into Practice of $\mathrm{C}$ Language Programming on Moodle. Project number: 2015 ZXJG 002.

\section{References}

[1] People's Government of Xixiu District. Implementation Plan of Pilot Demonstration Xixiu District of Anshun City in Guizhou Province [Z]. 2016-02.

[2] People's Government of Xixiu District. Work Plan of Pilot Demonstration Xixiu District of Anshun City in Guizhou Province [Z]. 2015-04.

[3] The Tunpu Mountains Jiangnan Leisure Tourism Resort [EB/OL].http://www.anshun.gov.cn/search.html, 2015-06/2017-06.

[4] Chen Min'er. Build E-commerce Industry of Guizhou Version by Taking Shortcut and Surpass with Full Efforts, [N]. Guizhou daily, 2015-08-21.

[5] Guo Yubin, Li Ximing. Application Research of On-line Teaching Platform for Computer Basics, Guizhou Daily [J], 2013, (04): 0065-02.

[6] Xiao Hui. Design and Implementation of On-line Platform for Computer Basics Teaching [J]. Computer Teaching, 2013, (15): 103-02.

[7] Qin Lu. Research on Remote Training Model for Farmers Based on Moodle Platform [D]. Jinan: Shandong Normal University, 2012.

[8] Wang Jun, Huang Shoumeng. Design and Implementation of Teaching Network Platform - Example of Computer Basic Teaching Website, [J]. Computer Knowledge and Technology, 2012, (30): 7248-02.

[9] Wang Huadong, Hu Haizhi. Design and Implementation of Basic Course of Computer Network [J] Public Science and Technology, 2006, (01): 0144-02.

[10] Lu Dayi. Construction and Application of On-line Teaching Platform of Computer Application Basic Course Based on Web [J]. Guangxi Light Industry, 2011, (01): 67-02. 